\title{
Bloqueio Peribulbar com Ropivacaína: Influência da Hialuronidase sobre a Qualidade do Bloqueio e a Pressão Intra-Ocular *
}

\section{Peribulbar Block with Ropivacaine: Effects of Hyaluronidase on Blockade Quality and Intraocular Pressure}

Paulo Sérgio Mateus Serzedo, TSA ${ }^{1}$, José Roberto Nociti, TSA ${ }^{2}$, Eduardo Barbin Zuccolotto, TSA ${ }^{1}$, Tatiana Lúcia Scalco ${ }^{3}$, Sérgio Borges Ferreira ${ }^{4}$

\section{RESUMO}

Serzedo PSM, Nociti JR, Zuccolotto EB, Scalco TL, Ferreira SB - Bloqueio Peribulbar com Ropivacaína: Influência da Hialuronidase sobre a Qualidade do Bloqueio e a Pressão Intra-Ocular

Justificativa e Objetivos - Alguns estudos têm relatado melhoria da qualidade do bloqueio peribulbar com o emprego de hialuronidase, enquanto outros concluem pela ausência de efeito. O objetivo deste estudo foi investigar a influência da hialuronidase sobre a pressão intra-ocular (PIO) e a qualidade do bloqueio peribulbar com ropivacaína a $1 \%$.

Método - Quarenta pacientes submetidos à cirurgia de catarata foram distribuídos de forma aleatória em dois grupos e submetidos a bloqueio peribulbar com $7 \mathrm{ml}$ de ropivacaína a $1 \%$ em técnica de dupla punção, com hialuronidase $50 \mathrm{UI} . \mathrm{ml}^{-1}$ no Grupo $A(n=20)$ e sem hialuronidase no Grupo $B(n=20)$. As medidas de PIO foram realizadas com tonômetro de aplanação de Perkins em quatro momentos: $M 0=$ antes do bloqueio (controle); $M 1=1 \mathrm{~min}$ após o bloqueio; $\mathrm{M2}=5 \mathrm{~min}$ após o bloqueio; $M 3=15$ min após o bloqueio. A qualidade foi avaliada pelo método de Nicoll, baseado na redução da motilidade do globo ocular.

Resultados - As médias de PIO $(\mathrm{mmHg})$ antes do bloqueio foram semelhantes nos dois grupos: 16,1 $\pm 2,1$ (A) vs 16,4 $\pm 3,3$ (B). Após o bloqueio, as médias de PlO foram significativamente menores no Grupo $A$ em relação ao Grupo $B$ nos três momentos: $M 1=11,7 \pm 2,4$ vs 17,9 $9 \pm 3,6 ; M 2=8,2 \pm 1,9$ vs $14,1 \pm 4,0 ; M 3=5,3 \pm 2,1$ vs $10,2 \pm 3,1$. O comportamento intragrupos também foi diferente. No Grupo $A$, as médias de PIO foram significativamente menores em relação ao controle nos três momentos após o bloqueio; no Grupo B a média de PIO elevou-se em M1 e foi significativamente inferior ao controle em M2 e M3. As médias para os índices de motilidade do globo ocular (Nicoll) foram significativamente menores no Grupo A em relação ao $B$ nos três momentos: $M 1=2,55$ vs 3,65; $M 2=$ 0,25 vs 2,$2 ; M 3=0,00$ vs 1,00 .

\footnotetext{
${ }^{*}$ Recebido do (Received from) CET/SBA da Santa Casa de Misericórdia de Ribeirão Preto (SCMRP), SP

1. Co-responsável pelo CET/SBA

2. Responsável pelo CET/SBA da SCMRP

3. $M E_{2}$ do CET/SBA da SCMRP

4. Oftalmologista Chefe do Instituto de Microcirurgia Ocular de Ribeirão Preto, SP
}

Apresentado (Submitted) em 28 de agosto de 2000

Aceito (Accepted) para publicação em 17 de outubro de 2000

Correspondência para (Mail to)

Dr. José Roberto Nociti

Rua Ayrton Roxo, 980

14025-270 Ribeirão Preto, SP

E-mail:carp@keynet.com.br

(c) Sociedade Brasileira de Anestesiologia, 2001
Conclusões - Quando se emprega solução de ropivacaína a $1 \%$ adicionada de hialuronidase 50 Ul. $\mathrm{ml}^{-1} \mathrm{em}$ bloqueio peribulbar, os valores da PIO são menores e a qualidade do bloqueio é melhor do que quando se utiliza ropivacaína a $1 \%$ sem hialuronidase.

UNITERMOS - ANESTÉSICOS, Local: ropivacaína; TÉCNICAS ANESTÉSICAS, Regional: peribulbar; TÉNICAS DE MEDIÇAO: pressão intra-ocular

\section{SUMMARY}

Serzedo PSM, Nociti JR, Zuccolotto EB, Scalco TL, Ferreira SB - Peribulbar Block with Ropivacaine: Effects of Hyaluronidase on Blockade Quality and Intraocular Pressure

Background And Objectives - Some studies have reported improved quality of peribulbar block by adding hyaluronidase to the local anesthetic solution while others claimed no beneficial effect. This study aimed at investigating the influence of hyaluronidase on intraocular pressure $(I O P)$ and the quality of peribulbar block with $1 \%$ ropivacaine.

Methods - Participated in this study 40 patients undergoing cataract surgery under peribulbar block who were randomly allocated to one of two groups according to the nature of the local anesthetic solution: Group $A(n=20), 1 \%$ ropivacaine $(7 \mathrm{ml})$ supplemented with $50 \mathrm{IU} \cdot \mathrm{ml}^{-1}$ hyaluronidase; Group $B(n=20)$, $1 \%$ plain ropivacaine $(7 \mathrm{ml})$. IOP measurements were performed by means of a Perkins applanation tonometer in four moments: $M 0$ = before block (control); $M 1$ = 1 min after block; M2 = 5 min after block; $M 3=15$ min after block. Quality was evaluated by Nicoll's method based on eye motility decrease.

Results - Mean IOP values $(\mathrm{mmHg})$ before block (MO) were similar for both groups: $16,1 \pm 2.1$ vs. $16.4 \pm 3.3$. After blockade, mean IOP values were significantly lower in Group $A$ as compared to Group $B$ in the three moments after block: $M 1=11.7 \pm$ 2.4 vs. $17.9 \pm 3.6 ; M 2=8.2 \pm 1.9$ vs. $14.1 \pm 4.0 ; M 3=5.3 \pm 2.1$ vs. $10.2 \pm 3.1$. IOP variations were also different within each group. In group $A$, mean values obtained in the three moments after block were significantly lower than control; in Group B, mean values significantly increased in $\mathrm{M} 1$ and were lower than control in M2 and M3. Mean eye motility scores were significantly lower in Group A as compared to Group B in M1 (2.55 vs. 3.65), M2 (0.25 vs. 2.2), and $M 3$ (0.00 vs.1.00).

Conclusions - When 1\% ropivacaine supplemented with 50 IU. $\mathrm{ml}^{-1}$ hyaluronidase is used in peribulbar block, IOP values are lower and blockade quality is significantly better than when $1 \%$ plain ropivacaine is used.

KEY WORDS - ANESTHETICS, Local: ropivacaine; ANESTHETIC TECHNIQUES, Regional: peribulbar; MEASUREMENT TECHNIQUES: intraocular pressure 


\section{INTRODUÇÃO}

$\mathrm{O}$ uso da ropivacaína em bloqueio peribulbar para cirurgia intra-ocular tem proporcionado bons resultados, combinando boa qualidade com baixa morbidade ${ }^{1-3}$. A hialuronidase é associada freqüentemente ao anestésico local neste tipo de bloqueio, com a finalidade de facilitar a difusão do mesmo graças à hidrólise das ligações glicosídicas no interior do ácido hialurônico que forma a barreira intersticial ${ }^{4}$. Alguns estudos têm relatado efetiva melhoria da qualidade do bloqueio com o emprego de hialuronidase ${ }^{5,6}$, enquanto outros têm concluído pela ausência de efeito ${ }^{7}$.

Por outro lado, já foi descrita diminuição da pressão intra-ocular (PIO) após bloqueio peribulbar com ropivacaína associada a hialuronidase ${ }^{8}$.

A finalidade deste estudo foi investigar a possível influência da hialuronidase sobre a qualidade do bloqueio obtido e a $\mathrm{PIO}$, após bloqueio peribulbar com ropivacaína.

\section{MÉTODO}

O estudo foi aprovado pela Comissão de Ética em Pesquisa do Hospital e dele fizeram parte 40 pacientes de ambos os sexos com idades entre 41 e 95 anos, estado físico ASA I, II ou III, encaminhados à cirurgia de catarata em regime ambulatorial. Foram excluídos portadores de glaucoma.

Não foi administrada medicação pré-anestésica e os pacientes foram distribuídos de forma aleatória em dois grupos, de acordo com a presença ou não de hialuronidase na solução de ropivacaína utilizada no bloqueio peribulbar:

Grupo A $(n=20)$ - Ropivacaína a $1 \%$ contendo hialuronidase 50 UI. $\mathrm{ml}^{-1}$

Grupo B $(n=20)$ - Ropivacaína a $1 \%$ sem hialuronidase

Foram monitorizados eletrocardiograma (ECG) contínuo, oximetria de pulso $\left(\mathrm{SpO}_{2}\right)$, pressão arterial sistólica (PAS), diastólica (PAD) e média (PAM), por método não invasivo. O bloqueio peribulbar foi realizado pelo método de dupla punção ${ }^{1}$, com agulha padrão $25 \times 6 \mathrm{~mm}$ (23 G-1), volume total de solução $7 \mathrm{ml}$, injetando-se $4 \mathrm{ml}$ através da pálpebra inferior na junção do terço lateral com os dois terços mediais da rima orbital, e $3 \mathrm{ml}$ através da pálpebra superior na borda súpero-interna da órbita. Após as injeções, foi aplicado baroftalmo sobre o olho fechado com peso de Mclntyre durante 10 minutos.

A medida da PIO foi realizada com tonômetro de aplanação de Perkins por observador independente (o próprio cirurgião) que desconhecia a natureza da solução, em quatro momentos:

$$
\begin{aligned}
& M 0=\text { Antes do bloqueio } \\
& M 1=1 \text { min após o bloqueio } \\
& M 2=5 \text { min após o bloqueio } \\
& M 3=15 \text { min após o bloqueio }
\end{aligned}
$$

A avaliação da qualidade do bloqueio foi feita nos momentos M1, M2 e M3 através do método descrito por Nicoll e col ${ }^{1,9}$, baseado na redução da motilidade do globo ocular, considerando-se como sinal de sucesso o índice igual ou inferior a 4.

Foi pesquisada a ocorrência de possíveis eventos adversos: dor à injeção do anestésico local, hipotensão arterial (diminuição da PA $>30 \%$ do controle), hipertensão arterial (aumento da PAS $>30 \%$ do controle), bradicardia (FC $\leq 50 \mathrm{bpm})$, taquicardia ( $F C \geq 120 \mathrm{bpm}$ ), disritmia cardíaca, náuseas e vômitos.

A comparação estatística entre as médias de idade e peso nos dois grupos foi feita pelo teste $t$ de Student; a comparação quanto ao sexo foi feita pelo teste do Qui-quadrado . A comparação das médias da PIO entre grupos e intragrupos foi feita pelo teste paramétrico de análise de variância two way. Para comparação das médias do índice de motilidade ocular (Nicoll) entre os grupos utilizou-se o teste Mann-Whitney e dentro de cada grupo a prova de Friedman. Em todos os testes o nível de significância adotado foi $5 \%(p<0,05)$.

\begin{tabular}{|c|c|c|c|}
\hline & & $\begin{array}{l}\text { Grupo A } \\
(n=20)\end{array}$ & $\begin{array}{l}\text { Grupo B } \\
(n=20)\end{array}$ \\
\hline \multirow[t]{2}{*}{ Idade (anos) } & Média \pm DP & $67,1 \pm 7,8$ & $69,1 \pm 10$ \\
\hline & Amplitude & $50-81$ & $41-81$ \\
\hline \multirow[t]{2}{*}{ Peso (kg) } & Média \pm DP & $70,9 \pm 13,4$ & $71,1 \pm 12,7$ \\
\hline & Amplitude & $48-96$ & $50-92$ \\
\hline \multicolumn{4}{|l|}{ Sexo } \\
\hline Masculino & & $9(45 \%)$ & $10(50 \%)$ \\
\hline Feminimo & & $11(55 \%)$ & $10(50 \%)$ \\
\hline
\end{tabular}

\section{RESULTADOS}

Os grupos foram homogêneos quanto aos dados demográficos, que estão apresentados na tabela I.

Afreqüência dos índices de motilidade ocular (Nicoll)em ambos os grupos nos vários momentos está expressa na tabela Il e suas médias na figura 1. Foram significativas as diferenças tanto entre os grupos como dentro de cada grupo.

Tabela II - Índices de Motilidade Ocular (Nicoll)

\begin{tabular}{lcccccc}
\hline & \multicolumn{2}{c}{ Grupo A (\% de pacientes) } & \multicolumn{3}{c}{ Grupo B (\% de pacientes) } \\
\hline Índice & M1 & M2 & M3 & M1 & M2 & M3 \\
\hline 0 & 5 & 75 & 100 & 5 & 30 & 45 \\
1 & 5 & 25 & 0 & 10 & 30 & 20 \\
3 & 40 & 0 & 0 & 20 & 30 & 30 \\
4 & 30 & 0 & 0 & 45 & 10 & 5 \\
5 & 20 & 0 & 0 & 20 & 0 & 0 \\
\hline
\end{tabular}

Comparação Estatística: Intragrupos (Friedman) - significante a partir de M2; Entre grupos (Mann-Whitney) - significante em todos os momentos 


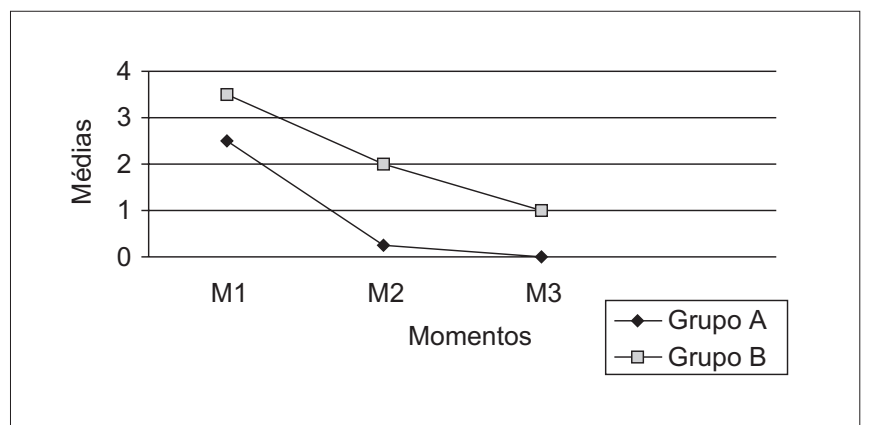

Figura 1 - Índice de Nicoll: Evolução das Médias

Os valores obtidos para as médias da PIO estão apresentados na tabela III e na figura 2. As diferenças foram estatisticamente significativas a partir do momento $\mathrm{M} 1$, tanto entre os grupos como dentro de cada grupo.

Tabela III - Medidas da Pressão Intra-Ocular (Média DDP)

\begin{tabular}{lcc}
\hline Momentos & Grupo A & Grupo B \\
\hline M0 & $16,1 \pm 2,1$ & $16,4 \pm 3,3$ \\
M1 & $11,7 \pm 2,4$ & $17,9 \pm 3,6^{*}$ \\
M2 & $8,2 \pm 1,9$ & $14,1 \pm 4,0^{*}$ \\
M3 & $5,3 \pm 2,1$ & $10,2 \pm 3,1^{*}$ \\
\hline
\end{tabular}

*Análise de variância "two way": diferença significante $(p<0,001)$

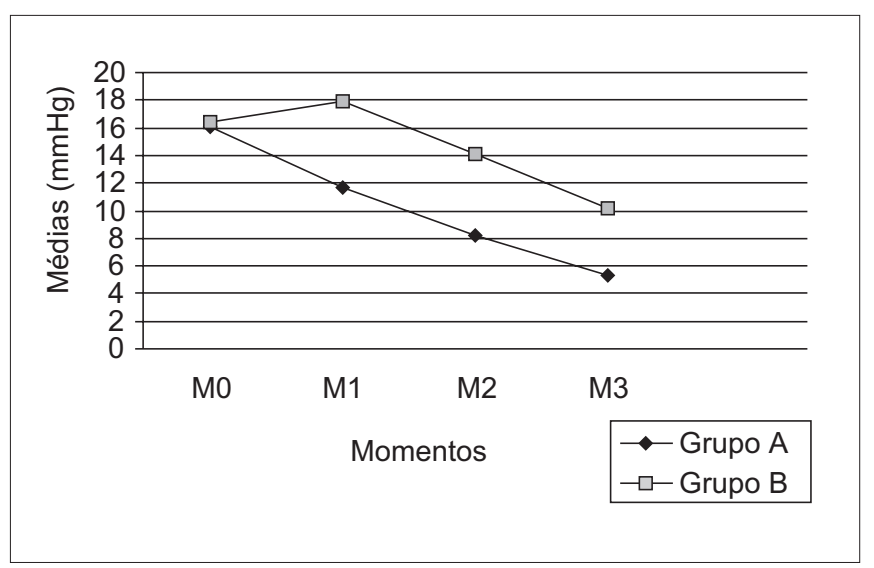

Figura 2 - Evolução das Médias da Pressão Intra-Ocular

Registrou-se queixa de dor à injeção da ropivacaína em quatro pacientes do Grupo B (20\%) e em um do Grupo A (5\%). Ocorreram episódios de hipertensão arterial em três pacientes do Grupo B (15\%) e em um do Grupo A (5\%). Houve necessidade de sedação (utilizou-se diazepam por via venosa) em um paciente de cada grupo (5\%). Não ocorreram outros eventos adversos.

\section{DISCUSSÃO}

Os resultados demonstraram bloqueio motor mais efetivo e melhor qualidade do bloqueio peribulbar quando a hialuronidase na concentração de $50 \mathrm{UI}^{\mathrm{m}} \mathrm{ml}^{-1}$ foi adicionada à solução de ropivacaína a $1 \%$. Por outro lado, a adição de hialuronidase influenciou também a variação da PIO. Nos pacientes submetidos ao bloqueio com ropivacaína adicionada de hialuronidase, registrou-se diminuição significativa da PIO em relação ao controle logo no primeiro minuto, acentuando-se nas medidas subseqüentes. Nos pacientes submetidos ao bloqueio com ropivacaína sem hialuronidase, houve elevação significativa da PIO no primeiro minuto, para depois ocorrer diminuição nas medidas seguintes. Mas os valores obtidos para as médias de PIO nos pacientes do Grupo A (com hialuronidase) foram significativamente menores do que os obtidos nos pacientes do Grupo B (sem hialuronidase), em todos os momentos após o bloqueio.

Estes resultados estão de acordo com observações de outros autores, que encontraram melhoria da qualidade do bloqueio peribulbar pela adição de hialuronidase à solução do anestésico local, utilizando concentrações da enzima similares ${ }^{10}$ ou mesmo inferiores ${ }^{11}$ às empregadas neste estudo.

Por outro lado, os valores mais elevados da PIO nos pacientes que não receberam hialuronidase foram detectados também por outros autores ${ }^{10}$, e esta ocorrência parece estar relacionada com a dispersão incompleta do anestésico local dentro do compartimento periocular. Sabe-se que o bloqueio peribulbar pode ocasionar elevação transitória da PIO devido ao aumento da pressão intraorbitária pela injeção do anestésico local. Entretanto, a PIO tende a retornar ao seu valor inicial devido à dispersão da solução e ao relaxamento da musculatura extraocular (secundário ao bloqueio), diminuindo a pressão externa sobre o globo ocular ${ }^{12}$.

É interessante salientar que, ao contrário do que ocorre com a bupivacaína ${ }^{10}$, a elevação inicial da PIO com a ropivacaína sem hialuronidase não se manteve nas medidas subseqüentes. Isto pode ser devido a um relaxamento mais completo da musculatura extraocular com a ropivacaína, já apontado em estudo anterior comparativo com a bupivacaína ${ }^{13}$. Além disso, é possível que a elevação inicial da PIO pela injeção do anestésico local seja contrabalanceada também por diminuição do volume sangüíneo intra-ocular secundário ao efeito vasoconstritor da ropivacaína ${ }^{14}$, fator inexistente com a bupivacaína, que é desprovida deste efeito.

Concluímos que, quando se emprega solução de ropivacaína a $1 \%$ adicionada de hialuronidase $50 \mathrm{UI}^{-1} \mathrm{ml}^{-1} \mathrm{em}$ bloqueio peribulbar, os valores da PIO são menores e a qualidade do bloqueio, avaliada pelo índice de motilidade do globo ocular é melhor do que quando se usa o mesmo anestésico local sem hialuronidase. A melhor dispersão da solução de anestésico local no compartimento periocular proporcionada pelo efeito característico da hialuronidase parece contribuir tanto para a diminuição da PIO como para facilitar o bloqueio das estruturas nervosas perioculares pelo agente anestésico. 


\section{Peribulbar Block with Ropivacaine: Effects of Hyaluronidase on Blockade Quality and Intraocular Pressure}

Paulo Sérgio Mateus Serzedo, M.D., José Roberto Nociti, M.D., Eduardo Barbin Zuccolotto, M.D., Tatiana Lúcia Scalco M.D., Sérgio Borges Ferreira, M.D.

\section{INTRODUCTION}

Ropivacaine for peribulbar block in intraocular surgeries has provided good results, combining high quality with low morbidity ${ }^{1-3}$. Hyaluronidase is commonly associated to local anesthetics in this type of blockade aiming at facilitating its spread thanks to the hydrolysis of glucoside bindings within the hyaluronic acid forming the interstitial barrier ${ }^{4}$. Some studies have reported a marked improvement in blockade quality with the use of hyaluronidase ${ }^{5,6}$ while others concluded for the absence of such effect ${ }^{7}$.

On the other hand, intraocular pressure (IOP) decrease after peribulbar block with ropivacaine associated to hyaluronidase has also been described ${ }^{8}$.

This study aimed at investigating the possible influence of hyaluronidase on blockade and IOP after peribulbar block with ropivacaine.

\section{METHODS}

After the Hospital Research Ethics Committee approval participated in this study 40 patients of both genders, aged 41 to 95 years, physical status ASAI, II or III, scheduled for outpatient cataract surgery. Patients with glaucoma were excluded.

No premedication was administered and patients were randomly distributed in two groups, according to the presence or not of hyaluronidase in the ropivacaine solution used for peribulbar block.

Group A $(n=20)-1 \%$ ropivacaine with 50 IU. $\mathrm{ml}^{-1}$ hyaluronidase

Group B $(n=20)$ - 1\% ropivacaine without hyaluronidase

Monitoring consisted of continuous ECG, pulse oximetry $\left(\mathrm{SpO}_{2}\right)$, non invasive systolic (SBP), diastolic (DBP) and mean blood pressure (MBP).

Peribulbar block was performed by the double puncture technique ${ }^{1}$ with a $25 \times 6 \mathrm{~mm}$ needle (23 G-1) in a total volume of 7 $\mathrm{ml}$ injecting $4 \mathrm{ml}$ through the lower eyelid in the junction of the lateral third with the two medial thirds of the orbital rime, and 3 $\mathrm{ml}$ through the upper eyelid in the upper-internal orbital border. After injection, an oculopression was applied on the closed eye with a Mcintyre weight for 10 minutes.

IOP was measured in four moments with a Perkins applanation tonometer by an independent observer (the surgeon himself) who did not know the solution's composition:
M0 - before blockade
M1 - 1 minute after blockade
M2 - 5 minutes after blockade
M3 - 15 minutes after blockade

Blockade quality was evaluated in moments $M 1, M 2$ and $M 3$ through the method described by Nicoll et al ${ }^{1,9}$ based on decreased eye motility, considering 4 or below a successful rate.

Possible adverse events were looked for: pain at local anesthetics injection, hypotension (below $30 \%$ of baseline), hypertension (above $30 \%$ of control), bradycardia ( $\mathrm{HR} \leq 50 \mathrm{bpm}$ ), tachycardia (HR $\geq 120 \mathrm{bpm})$, dysrhythmia, nausea and vomiting.

Student's $t$ test was used for statistical comparison between mean age and weight for both groups; Chi-square test compared gender. The two way analysis of variance parametric test was used to compare mean IOP between groups and within groups. To compare mean eye motility score (Nicoll) Mann-Whitney test was used between groups and Friedman test within each group. The level of significance of $5 \%$ ( $p<$ $0.05)$ was adopted for all tests.

\section{RESULTS}

Demographics data, which were homogeneous between groups, are shown in table I.

Table I - Demographics Data

\begin{tabular}{cccc}
\hline & & $\begin{array}{c}\text { Group A } \\
(\mathrm{n}=20)\end{array}$ & $\begin{array}{c}\text { Group B } \\
(\mathrm{n}=20)\end{array}$ \\
\hline Age (years) & Mean \pm SD & $67.1 \pm 7.8$ & $69.1 \pm 10$ \\
& Amplitude & $50-81$ & $41-81$ \\
Weight $(\mathrm{kg})$ & Mean \pm SD & $70.9 \pm 13.4$ & $71.1 \pm 12.7$ \\
& Amplitude & $48-96$ & $50-92$ \\
Gender & & & \\
\multicolumn{1}{c}{ Male } & & $9(45 \%)$ & $10(50 \%)$ \\
Female & & $11(55 \%)$ & $10(50 \%)$ \\
\hline
\end{tabular}

The frequency of eye motility scores (Nicoll) for both groups in all moments is shown in table II and their means in figure 1. Differences were significant both between and within groups.

Table II - Eye Motility Scores (Nicoll)

\begin{tabular}{lcccccc}
\hline & \multicolumn{2}{c}{ Group A (\% of patients) } & \multicolumn{2}{c}{ Group B (\% of patients) } \\
\hline Score & M1 & M2 & M3 & M1 & M2 & M3 \\
\hline 0 & 5 & 75 & 100 & 5 & 30 & 45 \\
1 & 5 & 25 & 0 & 10 & 30 & 20 \\
3 & 40 & 0 & 0 & 20 & 30 & 30 \\
4 & 30 & 0 & 0 & 45 & 10 & 5 \\
5 & 20 & 0 & 0 & 20 & 0 & 0 \\
\hline
\end{tabular}

Statistical Comparison: Within groups (Friedman) - significant as of M2; Between groups (Mann-Whitney) - significant in all moments 


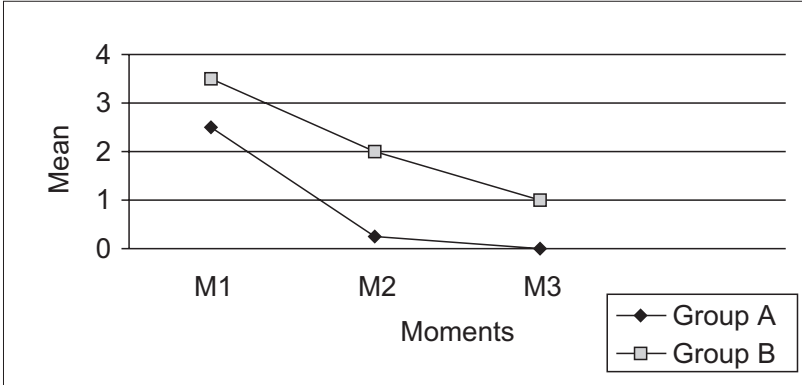

Figure 1 - Nicoll's Score: Mean Values Evolution

IOP mean values are shown in table III and figure 2. Differences were statistically significant as of M1 both between and within groups.

Table III - Intraocular Pressure Measurements (Mean \pm SD)

\begin{tabular}{lcc}
\hline Moments & Group A & Group B \\
\hline M0 & $16.1 \pm 2.1$ & $16.4 \pm 3.3$ \\
M1 & $11.7 \pm 2.4$ & $17.9 \pm 3.6^{*}$ \\
M2 & $8.2 \pm 1.9$ & $14.1 \pm 4.0^{*}$ \\
M3 & $5.3 \pm 2.1$ & $10.2 \pm 3.1^{*}$ \\
\hline
\end{tabular}

* "Two way" analysis of variance: significant difference $(p<0.001)$

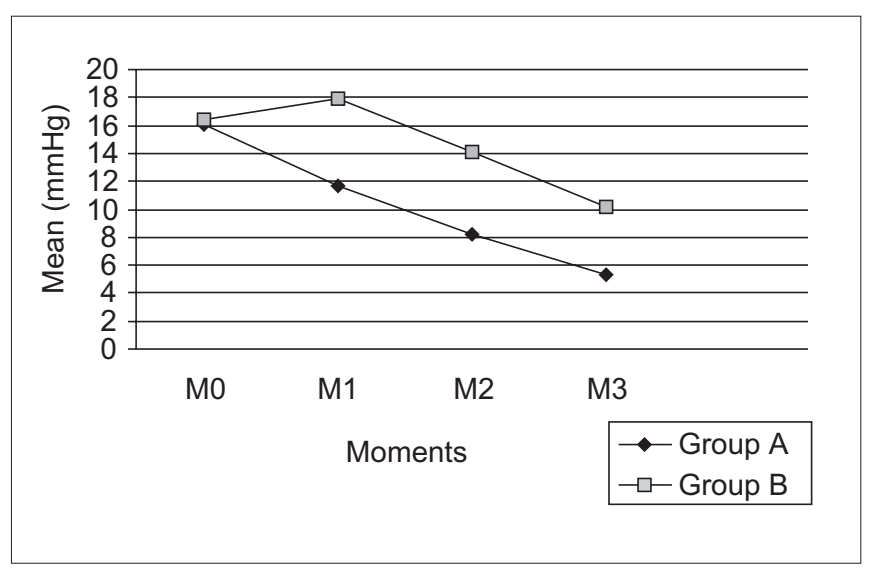

Figure 2 - Intraocular Pressure Mean Values Evolution

Four Group B (20\%) and one Group A (5\%) patients complained of pain at injection. Three Group B (15\%) and one Group A (5\%) patients had hypertension episodes. One patient of each group (5\%) needed sedation (intravenous diazepam). There were no other adverse effects.

\section{DISCUSSION}

Results have shown a more effective and better peribulbar block when $50 \mathrm{IU} \cdot \mathrm{ml}^{-1}$ hyaluronidase were associated to $1 \%$ ropivacaine. The addition of hyaluronidase has also influenced IOP variations. In patients submitted to the ropivacai- ne-hyaluronidase blockade, there has been a significant decrease in IOP as compared to the control group already in the first minute, which was more marked in subsequent measurements. In patients submitted to blockade with hyaluronidase-free ropivacaine, there has been a significant increase in IOP in the first minute which then decreased in subsequent measurements. But IOP means obtained for Group A patients (with hyaluronidase) were significantly lower as compared to Group B (without hyaluronidase) in all moments after blockade.

Such results are in line with other authors who observed a better peribulbar block with the addition of hyaluronidase to local anesthetics, using enzyme concentrations similar ${ }^{10}$ or even lower ${ }^{11}$ than those used in this study.

Higher IOP levels in patients not receiving hyaluronidase were also detected by other authors ${ }^{10}$, and this fact seems to be more related to the incomplete spread of local anesthetics within the periocular compartment. It is known that peribulbar block may cause a transient IOP increase due to the increase in intraorbital pressure caused by the anesthetic injection. However, IOP tends to return to its baseline value due to solution spread and extraocular muscles relaxation (secondary to blockade), thus decreasing external pressure on the eye 12

It is interesting to highlight that, as opposed to bupivacaine ${ }^{10}$, the initial IOP increase with hyaluronidase-free ropivacaine was not maintained in subsequent measurements. This may be due to a more complete extraocular muscles relaxation with ropivacaine already observed in a previous comparative study with bupivacaine ${ }^{13}$. Moreover, it is possible that the initial IOP increase caused by local anesthetics injection is further counterbalanced by a decrease in intraocular blood volume, secondary to the vasoconstrictor effect of ropivacaine ${ }^{14}$, which is not true for bupivacaine which lacks such effect. We concluded that when $1 \%$ ropivacaine is associated to 50 IU. $\mathrm{ml}^{-1}$ hyaluronidase for peribulbar block, IOP values are lower and blockade quality evaluated by eye motility score, is better than when the same local anesthetics is used without hyaluronidase. The better local anesthetic spread within the periocular compartment provided by the characteristic effect of hyaluronidase, seems to contribute both for IOP decrease and to facilitate periocular nervous structures blockade by the anesthetic agent.

\section{REFERÊNCIAS - REFERENCES}

01. Serzedo PSM, Nociti JR, Zuccolotto EB et al - Ropivacaína em bloqueio peribulbar: estudo comparativo com bupivacaína. Rev Bras Anestesiol, 1998;48:258-263.

02. Nicholson G, Sutton B, Hall GM - Ropivacaine for peribulbar anesthesia. Reg Anesth Pain Med, 1999;24:337-340.

03. Luchetti M, Magni G, Marraro G - A prospective randomized double-blind controlled study of ropivacaine $0.75 \%$ versus bupivacaine $0.5 \%$-mepivacaine $2 \%$ for peribulbar anesthesia. Reg Anesth Pain Med, 2000;25:195-200. 
04. Watson D - Hyaluronidase. Br J Anaesth, 1993;71:422-425.

05. Sarvela J, Nikki P - Hyaluronidase improves regional ophthalmic anaesthesia with etidocaine. Can J Anaesth, 1992;39:920-924.

06. Morsman CD, Holden R - The effects of adrenaline, hyaluronidase and age on peribulbar anaesthesia. Eye, 1992;6: 290-292.

07. Brydon CW, Basler M, Kerr WJ - An evaluation of two concentrations of hyaluronidase for supplementation of peribulbar anaesthesia. Anaesthesia, 1995;50:998-1000.

08. Serzedo PSM, Nociti JR, Zuccolotto EB et al - Pressão intraocular durante bloqueio peribulbar com ropivacaína a 1\%. Rev Bras Anestesiol, 2000;50:251-253.

09. Nicoll JM, Treuren B, Acharya PA et al - Retrobulbar anesthesia: the role of hyaluronidase. Anesth Analg, 1986;65:1324-1328.

10. Dempsey GA, Barrett PJ, Kirby IJ - Hyaluronidase and peribulbar block. Br J Anaesth, 1997; 78:671-674.

11. Kallio H, Paloheimo M, Maunuksela EL - Is hyaluronidase effective as an adjuvant in retrobulbar/peribulbar block? Internat Monitor, 1998;10:50.

12. Johnson RW, Forrest FC - Anaesthesia for Ophthalmic Surgery, em: Prys-Roberts C, Brown Jr BR - International Practice of Anaesthesia, Oxford, Butterworth Heinemann, 1996;110:1-29.

13. Serzedo PSM, Nociti JR, Zuccolotto EB et al - Pressão intraocular durante bloqueio peribulbar com ropivacaína ou bupivacaína: estudo comparativo. Rev Bras Anestesiol, 2000;50:341-344.

14. Ishiyama T, Dohi $\mathrm{S}$, lida $\mathrm{H}$ et al - The effects of topical and intravenous ropivacaine on canine pial microcirculation. Anesth Analg, 1997;85:75-81.

\section{RESUMEN}

Serzedo PSM, Nociti JR, Zuccolotto EB, Scalco TL, Ferreira SB - Bloqueo Peribulbar con Ropivacaína: Influencia de la Hialuronidasa sobre la Calidad del Bloqueo y la Presión Intra-Ocular

Justificativa y Objetivos - Algunos estudios han relatado mejoria de la calidad del bloqueo peribulbar con el uso de hialuronidasa, en cuanto otros han concluido por la ausencia del efecto. El objetivo de este estudio fue investigar la influencia de la hialuronidasa sobre la presión intra-ocular (PIO) y la calidad del bloqueo peribulbar con ropivacaína a $1 \%$.

Método - Cuarenta pacientes sometidos a cirugía de catarata fueron distribuidos de forma aleatoria en dos grupos $y$ sometidos a bloqueo peribulbar con $7 \mathrm{ml}$ de ropivacaína a $1 \%$ en técnica de dupla punción, con hialuronidasa $50 \mathrm{Ul} . \mathrm{ml}^{-1}$ en el Grupo $A(n=20)$ y sin hialuronidasa en el Grupo B $(n=20)$. Las medidas de PlO fueron realizadas con tonómetro de aplanación de Perkins en cuatro momentos: $M 0=$ antes del bloqueo (control); $M 1$ = 1 min después del bloqueo; $M 2=5 \mathrm{~min}$ después del bloqueo; M3 = 15 min después del bloqueo. La calidad fue evaluada por el método de Nicoll, fundamentado en la reducción de la motilidad del globo ocular.

Resultados - Las medias de PIO $(\mathrm{mmHg})$ antes del bloqueo fueron semejantes en los dos grupos: 16,1 $\pm 2,1$ (A) vs 16,4 \pm 3,3 (B). Después del bloqueo, las medias de PIO fueron significativamente menores en el Grupo A en relación al Grupo $B$ en los tres momentos: $M 1=11,7 \pm 2,4$ vs 17,9 $\pm 3,6 ; M 2=8,2 \pm$ 1,9 vs $14,1 \pm 4,0 ; M 3=5,3 \pm 2,1$ vs $10,2 \pm 3,1$. El comportamiento intragrupos también fue diferente. En el Grupo A, las medias de PIO fueron significativamente menores en relación al control en los tres momentos después del bloqueo; en el Grupo B la media de PIO se elevó en M1 y fue significativamente inferior al control en M2 e M3. Las medias para los índices de motilidad del globo ocular (Nicoll) fueron significativamente menores en el Grupo A en relación al $B$ en los tres momentos: $M 1=2,55$ vs 3,65; $M 2=0,25$ vs 2,2; $M 3=0,00$ vs 1,00 .

Conclusiones - Cuando se usa solución de ropivacaína a 1\% adicionada de hialuronidasa $50 \mathrm{UI}^{-1} \mathrm{ml}^{-1}$ en bloqueo peribulbar, los valores de la PIO son menores y la calidad del bloqueo es mejor de que cuando se utiliza ropivacaína a $1 \%$ sin hialuronidasa. 